\title{
La prise en compte du risque travailleurs dans la mise en œuvre du principe d'optimisation des doses au public
}

\author{
J. LOMBARD (*), A. OUDIZ (**), G. UZZAN (**)
}

\begin{abstract}
RÉSUMÉ
La mise en œuvre d'un système de traitement d'effluents visant à réduire les risques encourus par le public peut accroître le risque travailleurs du fait des operations d'exploitation et de maintenance associées à ce dispositif. On se trouve ainsi confronté à un problème de transfert de risque du public vers les travailleurs. Pour en tenir compte dans la procédure d'optimisation des doses délivrées au public, il convient de considérer simultanément le détriment pour le public et le détriment occasionné chez les travailleurs par les dispositifs de traitement à sélectionner.

On procède, dans le cas d'une centrale PWR, à l'optimisation des doses associées aux effluents liquides reçues par le public. On montre la variation des résultats en fonction de diverses hypothèses relatives aux valeurs de l'homme-sievert pour le public et les travailleurs.
\end{abstract}

\section{ABSTRACT}

The implementation of an effluent treatment system will reduce public radiological risk but it can increase the doses received by the workers in charge with the operation and the maintenance of the treatment system. Hence, we may have to deal with a trade-off between public and occupational risk. Consideration of public detriment as well as occupational detriment related to the treatment system will then be necessary in order to take into account this trade-off.

An example is considered here : optimization of effluent releases in a PWR plant. The variation of the results will be shown, according to different assumptions about man-sievert values for public and workers.

\section{INTRODUCTION}

Le système de limitation des doses recommandé par la CIPR dans sa publication 26 [1] repose sur trois principes :

a) la justification de la pratique (aucune pratique ne doit être adoptée à moins que son introduction ne produise un bénéfice net positif) :

(*) Centre d'étude sur l'évaluation de la protection dans le domaine nucléaire (CEPN), BP 48, 92260 Fontenay-aux-Roses.

(**) Association EURATOM/CEA-DPr, BP 6, 92260 Fontenay-aux-Roses. 
b) l'optimisation des expositions (toutes les expositions doivent être maintenues au niveau le plus bas que l'on pourra raisonnablement atteindre, compte tenu des facteurs économiques et sociaux) ;

c) le respect des limites de doses individuelles (l'équivalent de dose reçu par les individus ne doit pas dépasser les limites recommandées par la Commission dans les circonstances en question).

Lorsque la pratique est justifiée et que les limites de doses individuelles sont respectées, le système de limitation des doses se restreint à l'optimisation des expositions. Le principe consiste alors à mettre en balance les coûts de protection et le détriment sanitaire lié aux expositions. Dans certains cas, la réduction des expositions du public entraîne une exposition supplémentaire des travailleurs. II convient dès lors d'envisager le détriment sanitaire dans sa globalité : public et travailleurs.

\section{L'ÉTUDE DU TRANSFERT DANS LE CAS D'UN RÉACTEUR PWR}

\section{Les données considérées}

Ce phénomène de transfert de risque du public vers les travailleurs peut être illustré dans le cas du traitement des effluents d'un réacteur PWR. En effet, des opérations d'exploitation et de maintenance nécessaires au fonctionnement normal des différents systèmes de traitement des effluents (qui permettent la réduction des expositions du public) contribuent à l'exposition des travailleurs. II convient, dès lors, de tenir compte de cette surexposition des travailleurs dans la mise en œuvre du principe d'optimisation.

On peut estimer, à partir des résultats d'exploitation des réacteurs PWR de $900 \mathrm{MWe}$, que l'équivalent de dose collectif annuel travailleurs lié au fonctionnement annuel d'un PWR de $1300 \mathrm{MWe}$ est, en l'absence d'événements marquants tels que des défaillances importantes de composants irradiants nécessitant des arrêts de longue durée pour réparations, de 4 homme-Sv $\left(^{\star}\right)$. L'équivalent de dose collectif effectif annuel travailleurs dû aux systèmes de traitement des effluents actuellement retenus est d'environ le dizième de l'équivalent total soit 0,4 homme-Sv (cette estimation résulte d'une synthèse, réalisée avec l'exploitant, des données de diverses centrales PWR françaises).

Cette valeur est confirmée par une récente étude de l'Atomic Industrial Forum [2] qui situe l'équivalent de dose collectif effectif travailleurs imputable à l'ensemble des systèmes de traitement des effluents entre 0,4 et 0,6 homme-Sv/an.

Afin de pouvoir comparer plus finement les expositions du public et des travailleurs, il convient de décomposer par voie d'effluent l'équivalent de dose collectif effectif travailleurs lié aux différents systèmes de traitement. II est d'usage de différencier les systèmes de traitement selon les quatre voies suivantes : effluents gazeux, effluents liquides primaires, effluents liquides usés et effluents solides.

\footnotetext{
* 1 homme-sievert (homme-Sv) $=100$ hommes-rems.
} 
Les systèmes de traitement retenus actuellement pour chacune de ces voies sont les suivants :

a) pour les effluents gazeux : un stockage sous pression, durant un mois en moyenne, des effluents hydrogénés et un piégeage des iodes lors du balayage du bâtiment réacteur ;

b) pour les effluents liquides primaires: un stockage moyen de 10 jours suivi d'un passage sur déminéraliseur et évaporateur ;

c) pour les effluents liquides usés: un stockage moyen de 5 jours de l'ensemble des eaux usées suivi, pour les eaux les plus actives, d'un passage sur évaporateur thermique ;

d) pour les effluents solides (déchets) : l'enfûtage des résines des déminéraliseurs, des charbons des pièges à iode et des concentrats d'évaporateurs ainsi que le compactage des cartouches de filtre et des divers matériaux contaminés.

Les études rendant compte des doses collectives et individuelles reçues par les travailleurs lors des diverses opérations associées à l'exploitation d'une centrale nucléaire ne permettent pas, actuellement, d'établir avec précision la fraction de la dose collective travailleurs imputable à ces différents systèmes de traitement.

Nous avons donc estimé, en liaison avec l'exploitant, les équivalents de dose collectif effectif annuel travailleurs entraînés par le recours aux divers systèmes de traitement :
a) traitement des effluents gazeux
b) traitement des effluents primaires
c) traitement des effluents usés
d) traitement des effluents solides
0,01 homme-Sv/an ;
0,04 homme-Sv/an ;
0,15 homme-Sv/an;
0,20 homme-Sv/an .

L'équivalent de dose dû au traitement des effluents solides est dû, pour une part, aux manipulations attachées aux résidus du traitement des effluents primaires et usés. Les doses résulteraient ainsi pour moitié environ du compactage des divers matériaux contaminés (essentiellement des déchets divers issus des chantiers nucléaires) et pour moitié des résidus du traitement des effluents primaires et des effluents usés (concentrats issus des évaporateurs, des résines échangeuses d'ions, des filtres) ; seules, les doses résultant du conditionnement de cette deuxième moitié sont à prendre en compte dans cette étude.

En tenant compte de cette particularité, on peut estimer que les traitements des effluents primaires et usés entraînent des équivalents de dose collectifs effectifs annuels travailleurs respectivement de 0,1 et 0,2 homme-Sv/an.

Les équivalents de dose collectifs effectifs annuels travailleurs sont donc, en première approximation, les suivants (tableau I) :

TABLEAU I

L'exposition annuelle travailleurs imputable aux différentes voies d'effluents

\begin{tabular}{|l|c|}
\hline Voie d'effluent & Exposition annuelle des travailleurs \\
\hline Effluents gazeux & 0,01 homme-Sv/an \\
Effluents primaires & 0,1 homme-Sv/an \\
Effluents usés & 0,2 homme-Sv/an \\
Effluents solides & 0,1 homme-Sv/an \\
\hline
\end{tabular}


On constate que c'est le traitement des eaux usées qui contribue le plus au bilan dosimétrique des travailleurs. II est donc plus particulièrement intéressant d'envisager cette partie de l'installation. C'est pourquoi nous allons envisager l'optimisation des expositions imputables aux eaux usées.

Rappelons que l'optimisation pour une voie particulière consiste à rechercher parmi différents systèmes de traitement envisageables, celui qui conduit au meilleur compromis entre les coûts de protection et les expositions.

Ainsi a-t-on envisagé, outre le système actuellement retenu, noté TEU 2, ( 5 jours de stockage et passage sur évaporation thermique) deux systèmes moins performants vis-à-vis de la rétention des effluents et deux autres plus performants (voir tableau II).

TABLEAU ॥

Les systèmes de traitement des eaux usées envisagés

\begin{tabular}{|c|c|}
\hline $\begin{array}{c}\text { Système } \\
\text { de traitement }\end{array}$ & Caractéristiques \\
\hline TEU-0 & $\begin{array}{c}\text { Traitement minimal (collecte avec un simple stockage de transit } \\
\text { filtration). } \\
\text { TEU-1 }\end{array}$ \\
TEU-2 & 5 jours de stockage supplémentaires. \\
TEU-3 & 10 jours de stockage et évaporation thermique. \\
TEU-4 & 5 jours de stockage; évaporation thermique et déminéralisation \\
& en parallèle $\left(^{*}\right)$. \\
\hline
\end{tabular}

Le système de traitement conduisant au meilleur compromis est celui qui minimise la somme des coûts de protection $\mathrm{X}$ et du détriment $\mathrm{Y}$.

Le coût du détriment $Y$ est égal à la somme du coût du détriment public $Y_{p}$ et du coût du détriment travailleurs $Y_{t}$. $Y_{p}$ et $Y_{t}$ s'écrivent respectivement $Y_{p}=\alpha_{p} D_{p}$ et $Y_{t}=\alpha_{t} D_{t}$ où $\alpha_{p}$ et $\alpha_{t}$ désignent les coûts de l'homme-sievert public et travailleurs. $D_{p}$ est l'équivalent de dose collectif subsistant dans le public après le recours à un système de traitement, lequel entraîne pour les travailleurs un surcroît d'exposition égal à $D_{t}$ (exploitation et maintenance du système de traitement). La mise en œuvre du processus d'optimisation nécessite donc la connaissance des valeurs $X, D_{p}, D_{t}$ associées à chaque système de traitement et le choix de $\alpha_{p}$ et $\alpha_{\mathrm{t}}$.

(*) Une autre option, TEU-5, ne comportant que 5 jours de stockage et la déminéralisation pourrait être envisagée. En effet, dans des conditions favorables d'exploitation (taux de rupture de gaines faible), la déminéralisation est suffisante et la décontamination par l'évaporateur en série n'est pas indispensable.

Par contre, dans des conditions plus sévères de fonctionnement entraînant des activités plus élevées dans les effluents TEU, la présence d'un évaporateur peut présenter un intérêt certain, la déminéralisation ayant une efficacité moindre. Par ailleurs, les techniques actuelles de déminéralisation ne permettent pas de traiter certains effluents chimiquement chargés (par exemple, les solutions de décontamination en provenance des ateliers chauds). 
Le coût $X$ de chaque système a été calculé sous forme de coût total actualisé sur 20 ans d'exploitation de la centrale nucléaire. II prend en compte les coûts d'investissements directs (construction et montage des équipements) et indirects (frais de préexploitation et intérêts intercalaires), ainsi que les coûts d'exploitation et de maintenance. Les valeurs, regroupées dans le tableau III, sont issues d'une étude récente [3].

L'équivalent de dose collectif effectif pour le public $D_{p}$ associé à chacun des systèmes a été déterminé pour 20 ans d'exploitation de la centrale à partir des activités rejetées. L'évaluation fait intervenir des modèles de diffusion, de consommation et de population. Le calcul a été effectué dans un rayon de $100 \mathrm{~km}$ autour d'une centrale située en bord de fleuve. Les valeurs, regroupées dans le tableau III, sont issues de la même étude [3].

L'équivalent de dose collectif effectif travailleurs $D_{t}$ associé à chaque système a été estimé à partir de la valeur déterminée pour le système de référence TEU-2 $=0,2$ homme-Sv/an. On a fait l'hypothèse que TEU-0 entraîne une dose collective nulle, TEU-1 conduirait à une valeur de 0,05 homme-Sv/an, TEU-3 conduirait à une valeur pratiquement identique à celle de TEU-2 et que TEU-4 conduirait à une valeur plus faible, 0,1 homme-Sv/an $\left(^{\star}\right)$, ce système étant comparable à celui actuellement retenu pour le traitement des effluents primaires. Afin de rendre comparable $D_{t}$ à $D_{p}$ et $X$, ces diverses valeurs ont été multipliées par 20, nombre d'années d'exploitation envisagé. Les valeurs sont regroupées dans le tableau III.

TABLEAU III

Les données pour 20 ans de fonctionnement normal d'une centrale PWR de 1300 MWe

\begin{tabular}{|c|c|c|c|}
\hline \multirow{2}{*}{$\begin{array}{c}\text { Système de } \\
\text { traitement }\end{array}$} & $\begin{array}{c}\text { Coût } X \\
\left(10^{6} \mathrm{FF}-1980\right)\end{array}$ & $\begin{array}{c}\text { Equivalent de dose collectif effectif } \\
\text { (homme-Sv) }\end{array}$ & $\begin{array}{c}\text { Travailleurs } \mathrm{D}_{\mathrm{t}} \\
\text { (homme-Sv) }\end{array}$ \\
\cline { 3 - 4 } & 0 & 8 & 0 \\
\hline TEU-0 & 13,2 & 5,8 & 1 \\
TEU-1 & 25,9 & 0,086 & 4 \\
TEU-2 & 29,7 & 0,045 & 4 \\
TEU-3 & 28,7 & 0,006 & 2 \\
TEU-4 & & & \\
\hline
\end{tabular}

\section{La procédure d'optimisation}

On peut remarquer d'emblée que TEU-3 n'est pas performant vis-à-vis de TEU-4, son coût étant supérieur $(29,7$ contre 28,7$)$ de même que les équivalents de dose collectifs effectifs $(0,045$ contre 0,006 homme-Sv pour le public et 4 contre 2 homme-Sv pour les travailleurs). Nous n'envisageons donc pas TEU-3 lors du processus d'optimisation. Les valeurs $X, D_{p}$ et $D_{t}$ étant déterminées, il convient de fixer $\alpha_{\mathrm{p}}$ et $\alpha_{\mathrm{t}}$. Les valeurs de l'homme-rad évoquées dans la recommandation 22 de la CIPR [4] sont comprises entre

$\left.{ }^{\star}{ }^{\star}\right)$ La déminéralisation serait utilisée de préférence à l'évaporation et les travailleurs seraient de ce fait moins exposés qu'avec TEU-2. 
10 et 250 dollars. A la même époque, la NRC proposait 1000 dollars par homme-rem pour le public [5]. Tout récemment, le NRPB a envisagé plusieurs valeurs de l'homme-sievert public comprises entre 2000 et 500000 livres [6]. En résumé, on trouve une fourchette de valeurs comprises entre 10 et 1000 dollars l'homme-rem soit $(1000-100000) \$$ l'hommesievert. Ces différentes valeurs, même 100000 dollars $\left(\simeq 0,5.10^{6} \mathrm{FF}\right)$ l'homme-sievert, conduisent comme nous le verrons ultérieurement au choix de TEU-O (ne rien faire), que l'on prenne en compte ou non le risque travailleurs. Ceci traduit le fait que les mesures de protection du public actuellement retenues pour les réacteurs PWR correspondent à des valeurs de $\alpha$ supérieures à $0,5.10^{6} \mathrm{FF}$. En effet, si l'on examine les systèmes retenus actuellement, dont (TEU-2), les valeurs d' $\alpha_{p}$ qui leur correspondent $\left(\frac{X}{D_{p}}\right)$ sont bien supérieures (entre $3.10^{6}$ et $\left.6.10^{6} \mathrm{FF}\right)$ [7]. II en va de même pour $\alpha_{\mathrm{t}}$, les mesures retenues actuellement pour la protection des travailleurs correspondant à des valeurs d' $\alpha_{t}$ souvent supérieures (entre $3.10^{4}$ et $3.10^{6} \mathrm{FF}$ [8].

C'est pourquoi, pour la première analyse, nous retiendrons pour $\alpha_{\mathrm{p}}$ une valeur supérieure à celles évoquées dans la littérature : $5.10^{6} \mathrm{FF}$. Nous admettrons également, dans un premier temps, que la radioprotection du public et celle des travailleurs sont jugées d'importance égale et, par conséquent, nous adopterons également $5.10^{6} \mathrm{FF}$ pour la valeur de $\alpha_{t}$ travailleurs $\left(^{\star}\right)$. Nous modifierons, par la suite, les valeurs d' $\alpha_{p}$ et $\alpha_{t}$ dans le cadre de l'analyse de sensibilité.

Nous obtenons, pour chaque système, les valeurs de $\mathrm{X}+\alpha_{\mathrm{p}} \mathrm{D}_{\mathrm{p}}+\alpha_{\mathrm{t}} \mathrm{D}_{\mathrm{t}}$ suivantes (tableau IV).

TABLEAU IV

Détermination de $\mathbf{X}+\mathrm{Y}_{\mathrm{p}}+\mathrm{Y}_{\mathrm{t}}$ dans le cas où $\alpha_{\mathrm{p}}=\alpha_{\mathrm{t}}=5.10^{6} \mathrm{FF}$

\begin{tabular}{|c|c|c|c|c|}
\hline \multirow[b]{2}{*}{$\begin{array}{l}\text { Système de } \\
\text { traitement }\end{array}$} & \multirow[b]{2}{*}{$\begin{array}{l}\text { Coût X } \\
\left(10^{6} \mathrm{FF}\right)\end{array}$} & \multicolumn{2}{|c|}{ Détriment } & \multirow{2}{*}{$\begin{array}{c}X+\alpha_{p} D_{p}+\alpha_{t} D_{t} \\
\left(10^{6} F F\right)\end{array}$} \\
\hline & & $\begin{array}{c}\text { Public } \alpha_{p} D_{p} \\
\left(10^{6} \mathrm{FF}\right)\end{array}$ & $\begin{array}{c}\text { Trav. } \alpha_{\mathrm{t}} \mathrm{D}_{\mathrm{t}} \\
\left(10^{6} \mathrm{FF}\right)\end{array}$ & \\
\hline $\begin{array}{l}\text { TEU-0 } \\
\text { TEU-1 } \\
\text { TEU-2 } \\
\text { TEU-4 }\end{array}$ & $\begin{array}{c}0 \\
13,2 \\
25,9 \\
28,7\end{array}$ & $\begin{array}{l}40 \\
29 \\
0,43 \\
0,03\end{array}$ & $\begin{array}{r}0 \\
5 \\
20 \\
10\end{array}$ & $\begin{array}{l}40 \\
47,2 \\
46,33 \\
38,73\end{array}$ \\
\hline
\end{tabular}

C'est le système de traitement TEU-4 qui, tout en assurant la meilleure protection du public (sans pour autant occasionner l'exposition maximale des travailleurs), minimise la somme des coûts de protection et du détriment dans le cas où $\alpha_{p}$ et $\alpha_{t}$ valent 5.106 FF.

Ces résultats dépendent essentiellement des choix d' $\alpha_{\mathrm{p}}$ et $\alpha_{\mathrm{t}}$. Etant donné que la question de la détermination de la valeur de l'homme-sievert est un élément central du processus d'optimisation et qu'il n'existe pas de consensus quant à la valeur à retenir, il convient de procéder à une analyse de sensibilité afin de tester la variabilité des résultats aux choix d' $\alpha_{\mathrm{p}}$ et $\alpha_{\mathrm{t}}$.

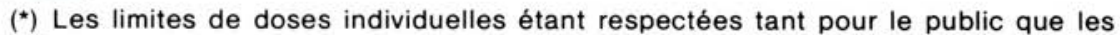
travailleurs, nous ne prenons pas en compte, conformément à la CIPR 26, la distribution des doses public et travailleurs. 
On peut envisager de modifier l'importance relative d' $\alpha_{p}$ et $\alpha_{t}$ ou de changer la valeur commune de référence. Nous envisageons ici les cas suivants :

1) Non prise en compte du risque travailleurs $\left(\alpha_{\mathrm{p}}=5.10^{6} \mathrm{FF} ; \alpha_{\mathrm{t}}=0\right)$.

2) Prépondérance accordée au risque public ( $\alpha_{p}=5.10^{6} \mathrm{FF} ; \alpha_{t}=0,5.10^{6} \mathrm{FF}$ ).

3) Prépondérance accordée au risque travailleurs $\left(\alpha_{p}=0,5.10^{6} \mathrm{FF}\right.$; $\alpha_{\mathrm{t}}=5.10^{6} \mathrm{FF}$.

4) Réduction de la valeur de référence d' $\alpha\left(\alpha_{\mathrm{p}}=\alpha_{\mathrm{t}}=0,5 \cdot 10^{6} \mathrm{FF}\right)$.

La somme des coûts de protection et du détriment pour chacun des systèmes de traitement évolue alors comme suit (tableau V).

TABLEAU V

Détermination de $X+Y_{p}+Y_{t}$ pour les différents cas envisagés

\begin{tabular}{|c|c|c|c|c|c|}
\hline $\begin{array}{l}\text { Système de } \\
\text { traitement }\end{array}$ & $\begin{array}{c}\text { Référence } \\
\alpha_{p}=5.10^{6} \\
\alpha_{\mathrm{t}}=5.10^{6}\end{array}$ & $\begin{array}{c}\text { Analyse } 1 \\
\alpha_{\mathrm{p}}=5.10^{6} \\
\alpha_{\mathrm{t}}=0\end{array}$ & $\begin{array}{c}\text { Analyse } 2 \\
\alpha_{p}=5.10^{6} \\
\alpha_{t}=0,5.10^{6}\end{array}$ & $\begin{array}{c}\text { Analyse 3 } \\
\alpha_{p}=0,5.10^{6} \\
\alpha_{t}=5.10^{6}\end{array}$ & $\begin{array}{c}\text { Analyse } 4 \\
\alpha_{p}=0,5 \cdot 10^{6} \\
\alpha_{t}=0,5 \cdot 10^{6}\end{array}$ \\
\hline $\begin{array}{l}\text { TEU-0 } \\
\text { TEU-1 } \\
\text { TEU-2 } \\
\text { TEU-4 }\end{array}$ & $\begin{array}{l}40 \\
47,2 \\
46,33 \\
\left.38,73 \quad{ }^{*}\right)\end{array}$ & $\begin{array}{l}40 \\
42,2 \\
26,33\left(^{\star}\right) \\
28,73\end{array}$ & $\begin{array}{l}40 \\
42,7 \\
28,33\left(^{\star}\right) \\
29,73\end{array}$ & $\begin{array}{l}4 \\
21,1 \\
45,94 \\
38,7\end{array}$ & $\begin{array}{l}4 \\
16,6 \\
27,94 \\
29,7\end{array}$ \\
\hline
\end{tabular}

De ce tableau, on déduit que si le détriment public est peu valorisé $\left(\alpha_{p}=0,5.10^{6}\right)$, le système minimisant $X+Y_{p}+Y_{t}$ est TEU-0; si le détriment public est fortement valorisé $\left(\alpha_{\mathrm{p}}=5.10^{6}\right)$ avec un détriment travailleurs peu valorisé $\left(0\right.$ ou $\left.0,5.10^{6}\right)$, le système minimisant $X+Y_{p}+Y_{t}$ est TEU-2 $\left({ }^{\star \star}\right)$ et si les deux détriments sont fortement valorisés, le système minimisant $X+Y_{p}+Y_{t}$ est TEU-4.

\section{CONCLUSION}

La prise en compte du risque travailleurs modifie donc le résultat de l'étude de l'optimisation des expositions du public liées au fonctionnement d'un réacteur. Notons, tout d'abord, que si les données dosimétriques travailleurs concernant la contribution des systèmes de traitement sont entachées d'une certaine incertitude, compte tenu de l'information disponible actuellement, elles ne sont pas moins issues de données d'observation, alors que les doses au public proviennent d'une évaluation par modèle de transfert. (II convient, cependant, de noter que les données sur lesquelles sont fondées ces estimations théoriques sont elles-mêmes basées sur les bilans de rejets des réacteurs en exploitation). Ceci étant précisé, le problème posé par le transfert du risque public-travailleurs mérite, selon nous, d'être pris en considération. II serait dès lors opportun de procéder à l'examen des systèmes de traitement des effluents d'autres installations du cycle du combustible dans la perspective de ce transfert.

$\left.{ }^{\star}\right)$ Minimum de $X+Y_{p}+Y_{t}$.

(**) Les écarts entre TEU-2 et TEU-4 étant faibles dans ces deux cas, il semble plus réaliste de considérer ces deux systèmes comme équivalents. 
A cet égard on doit noter que la nécessité de déterminer la performance des systèmes de traitement, de recenser les diverses variantes possibles, apparaîtra de plus en plus clairement à l'avenir dans le contexte réglementaire de l'application du nouveau système de limitation des doses.

La connaissance, même approximative, des performances des systèmes de traitement au stade de la conception des installations nucléaires favorisera probablement l'innovation technologique et contribuera à la recherche de dispositifs plus aptes à résoudre simultanément les problèmes de radioprotection du public et des travailleurs.

\section{RÉFÉRENCES}

[1] COMMISSION INTERNATIONALE DE PROTECTION RADIOLOGIQUE. Recommandations de la Commission Internationale de Protection Radiologique (adoptées le 17 janvier 1977), Publication CIPR n²6, Pergamon Press, 1980. Version anglaise : Oxford: Pergamon Press, 1977.

[2] Study of the effects of reduced occupational radiation exposure limits on the nuclear power industry. Rapport de l'Atomic Industrial Forum, AIF/NES-P-017, 1980.

[3] LOCHARD J., MACCIA C., PAGES P. Un exemple d'évaluation de la protection radiologique du public : le cas du traitement des effluents radioactifs d'une centrale nucléaire à eau préssurisée en fonctionnement normal. Rev. Epidémiol. Santé Publ. $1982,30,246-263$.

[4] COMMISSION INTERNATIONALE DE PROTECTION RADIOLOGIQUE. Les implications des recommandations de la Commission de maintenir les doses aux valeurs les plus faibles qu'il soit possible d'atteindre sans difficulté, Rapport du Comité 4 de la CIPR adopté par la Commission en avril 1973, Publication CIPR no 22. CEA, Département de Protection, 1974. Version Anglaise : Oxford: Pergamon Press, 1973 .

[5] UNITED STATES NUCLEAR REGULATORY COMMISSION, 10 CFR Part 50. Appendix I Numerical guides for design objectives and limiting conditions for operation to meet the criterion "As low as practicable" for radioactive materials in light water cooled power reactor effluents. Fed. Reg., 1975, 40, 19442-19443; $40818 ; 58847$.

[6] CLARK M.J., FLEISHMAN A.B., WEBB G.A.M. Optimisation of the radiological protection of the public. NRPB - R 120, 1981.

[7] LOCHARD J., MACCIA C., PAGES P. Application du principe d'optimisation de la CIPR à la comparaison de systèmes de traitement des effluents d'un réacteur PWR (IAEA-SM 258/28). In : Application of the dose limitation system in nuclear fuel cycle facilities and other radiation facilities, Madrid, 19-23 Oct. 1981 (à paraitre).

[8] LOCHARD J., MACCIA., PAGES P. La valeur implicite de I'homme-rem travailleur. Rapport CEPN n 45, 1981. 